$\begin{array}{cc}t \text { in Miniten } & i \text { in Ampere } \\ 0 & 0,137 \\ 4 & 0,117 \\ 20 & 0,082 \\ 41 & 0,067\end{array}$

Un die Abscheidung des Natriums rascher zu gestalten, wurde hierbei von Zeit zu Zeit $1 \mathrm{ccm} 1 / 10 \mathrm{n} \mathrm{HCl}$ zur Lösung gefügt. Doch muß vermieden werden, den Neutralitätspunkt zu überschreiten, da sonst Bildung von Wolframblas stattindet. Das nach 45 Minuten gebildete Sol war in nicht zu dicken Schichten klar durchsichtig und tief schwarzbraun gefärbt; in verdünnter Lösung ist es gelbbraun. Zusatz von $\mathrm{KCl}$ flockt einen schwarzen Körper aus, der dem niederen Oxyd ähnelt, das ich bei Abscheidungsversuchen von Wolfram aus angesäuerten Perwolframatlösungen an der Platinkathode erhielt. Man bekommt sogar dasselbe Sol ... in der Durchsicht nicht zu unterscheiden, dagegen im auffallenden Licht grau -, wenn man die Abscheidung des Oxyds durch Ueberziehen der Kathode mit einem Kautschukdiaphragma verhindert. Doch ist es dann bereits wenige Stunden nach der Herstellung geflockt, während das in der Hildebrandzelle hergestellte erst nach 3 Wochen einen Teil ausfallen Jäßt. Daß bei dem vorhandenen großen Alkaligehalt, überhaupt zunächst einige Stabilität vorhanden ist, glaube ich der schützenden Wirkung der Harzbestandteile oder etwaiger Hydrierungsprodukte des Kautschuks zuschreiben $z \mathfrak{u}$ müssen, da man nach der Flockung des Oxyds auch organische Sedimente wahrnimmt und auch während und nach der Elektrolyse ein aromatischer Geruch bemerk. bar ist.

Leipzig. Physikalisch-chemisches Institut.

\title{
Einfluß der Wolframsäure auf die Gelatinierung der Kieselsäure in stark salzsaurer Lösung.
}

Von M. Kröger (Leipzig).

Die Untersuchung bestand in einer Zugabe von konzentrierter Salzsäure zu einem Gemisch von Wasserglas- und Natriumwolframatlösung und Beobachtung der Dauer der Gelatinierungszeit. Infolge der angewandten MeBmethode war die Aufstellung des gesamten Diagramms von 100 Proz. $\mathrm{SiO}_{2}, 0$ Proz. $\mathrm{WO}_{3}$ bis 0 Proz. $\mathrm{SiO}_{2}, 100$ Proz. WO 3 nicht möglich, sondern es konnte nur der linke Teil Berücksichtigung finden.

Im Gegensatz zu anderen Zusätzen zeigte die Wolframsäure bereits in geringer Konzentration beträchtliche Verkürzung der Gelatinierungszeit, und ich wollte diese Tatsache analytisch - nötigenfalls viskosimetrisch - nutzbar machen. Bei der genauen Verfolgung ergab sich jedoch ein Kurvenverlauf, der diese Verwendung wenigstens zum Teil unmöglich macht. Daneben zeigten sich überraschende Trübungserscheinungen.

Ausführung der Versuche: Es wurd dabei die Konzentration des Wasserglases und der Salzsäure immer gleich gehalten und nur eine Zugabe von Wasser in wachsendem Maße durch Wolframatlösung ersetzt. Die Vermischung geschah in einem mit eingeschliffenem Stöpsel versehenen Reagenzrohr, das sich in zwei Schenhel teilte (Fig. 1).

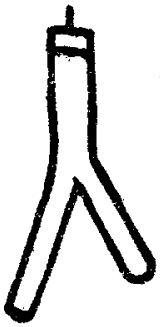

Fig. 1
(Eingegangen am 11. Oktober 1921.

Die Lösungen wurdent aus Büretten mit der dabei möglichen $\mathrm{Ge}$ nauigkeit zufließen gelassen, und zwar die Wasserglas- ind die Wolframatlösung und das Wasser in den einen Schenkel, die Salzsäure in den andern. Beim Vermischen tritt unter diesen Verhältnissen keine Abscheidung von $\mathrm{SiO}_{2}$ und $\mathrm{WO}_{3}$ ein. Nach dem Vermischen wurde die Lösung in Reagenzrohre um-

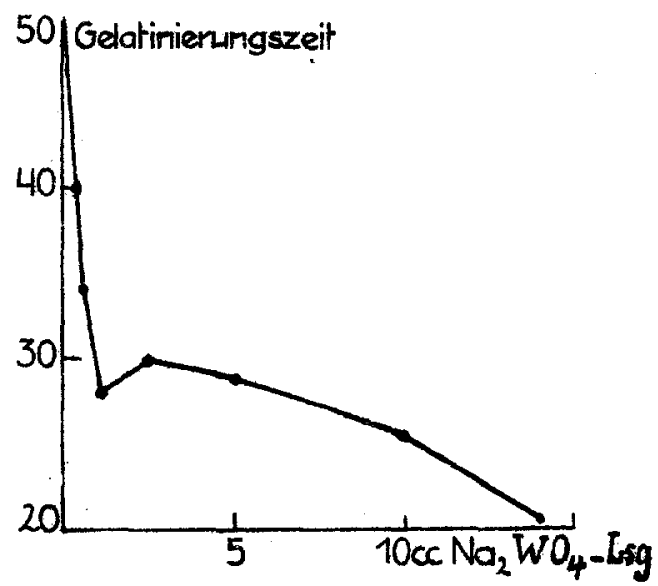

Fig. 2 
gefüllt. Da die Gelatinierungszeit mit zunehmender Temperatur stark verkürzt wird, war es nötig, den Einflub der Wärmetönung aufzuheben. Dies geschah in der Weise, dab die Reagenzrohre mit der Lösung sogleich in einen Thermostaten von $10^{\circ}$ höherer Temperatur, als die Zimmertemperatur betrug, eingetaucht wurden. Es hatte sich gezeigt, dab die Erhöhung der Temperatur etwa dieser Größle entsprach. Der geringe EinfluB der Wolframsäure auf die Wärmetönung - es handelt sich um 1 bis $2^{\circ}$ in den größten Zugaben ‥ blieb unberücksichtigt. Als fertig gelatiniert wurde die Lösung angesehen, bei der die Oberfläche beim Umkippen gerade noch eine schwache Krümmung aufwies. Dieser Zustand ist bei den angegebenen Zeiten auf eine viertel Minute genau reproduzierbar.

Gehalt der Lösungen :

Wasserglasiösung $33,7 \mathrm{Proz}$. Si $\mathrm{O}_{2}$-Gehalt Salzsäure $9,77 \mathrm{n}$

Natriumwolframatlosung 10,0 Proz. $\mathrm{Na}_{2} \mathrm{WO}_{4}, \mathrm{H}_{2} \mathrm{O}$

Die Ergebnisse finden sich in Tabelle I und Figur 2.

Die Kurve der Gelatinierungszeiten weist also ein deutliches Minimum und Maximum
Tabelle l.

$\grave{t}=29,0^{\circ} ;$ Luft $19,0^{\circ}$.

\begin{tabular}{|c|c|c|c|c|}
\hline \multicolumn{4}{|c|}{ ccm der verwendeten Lösungen: } & \multirow{2}{*}{$\begin{array}{c}\text { Gelatinie } \\
\text { rungszeit } \\
\text { in } \mathrm{Min} .\end{array}$} \\
\hline $\mathrm{HCl}$ & $\mathrm{Na}_{2} \mathrm{WO}_{4}$ & $\mathrm{H}_{2} \mathrm{O}$ & $\mathrm{SiO}_{2}$ & \\
\hline 1. 9,00 & $\ldots$ & & 70 & 49 \\
\hline 2. 9,00 & 0,5 & 14 & 7, & 34 \\
\hline 3. 9,00 & 1,0 & 14, & 7, & 28,0 \\
\hline 4. 9,00 & 2,5 & 12 & 7, & 30,0 \\
\hline 9,0 & 5,0 & 10,0 & 7,00 & 29,0 \\
\hline 9,00 & 10,0 & 5,0 & 7,6 & 25,5 \\
\hline 9,0 & 15,0 & - & 7,00 & 18,0 \\
\hline
\end{tabular}

auf. Von Versuch 4 an sind die Lösungen bereits beim Vermischen deutlich gelb gefärbt. Sie trüben sich im Laufe der folgender Tage deutlich gelb; 4 und 5 werden zunächst grün; offenbar eine Mischfarbe des Gelb mit der bläulichen Opaleszenz der Kieselsäuregele: in 6 und 7 scheint die Wolframsäure sehr bald grobdispers ausgeschieden zu werden. Dagegen unterscheiden sich 1 und 3 äuberlich in nichts, wohingegen 2 nach etwa einem Tage stark weib getrübt wird.

Leipzig.

Physikalisch-chemisches institu.

\section{Sichtbarmachung einzelner Submikronen im Tyndallkegel mit bloBem Auge.}

Von J. Traube und P. Kle in (Charlottenburg).

(Fingegangen aro 13, Oktober 1921.

Im Laufe von Versuchen, die mit Hilfe des Tyndallkegels ausgeführt wurden, beobachteten wir bei Zusatz von wenig Natriumkarbonat zu einer bestimmten Bleinitratlösung eine höchst eigenartige Erscheinung. In der bis dahin fast optisch leeren Lösung entstand ein Bleikarbonatkegel, der im Gegensatz zum gewöhnlichen auch mit bloBem Auge auflösbar war.

Den Kegel erzeugten wir mit einer Mikrobogenlampe und einem Spalt aus Pappe. Die Versuche wurden in einem Glasgefäbe mit planparallelen Wänden vorgenommen. Die Beobachtung erfolgte senkrecht zum Lichtstrahl.

Der Verlauf des Versuches ist folgender:

$\mathrm{Zu} 30 \mathrm{ccm} 1 / 30000 \mathrm{n}$ Bleinitratlösung werden zwei Tropfen (aus einem T.K.-Tropfglas) $1 / 2 n$ Natriumkarbonatlösung zugesetzt und sogleich ein wenig umgeschüttelt. Es bildet sich hierbei allmählich ein aus Bleikarbonat be stehender schwacher Kegel. Wenn man denselben mit einer Lupe beobachtet, so sieht man nach etwa 40 bis 60 Sskunden eine auberordentlich grobe Anzahl kleiner Teilchen, die sich in heftiger vollstänarg unregelmäbiger Bewegung befinden. Man hat etwa den Eindruck eines herumschwirrenden Mückenschwarmes. Die Lichtstärke, die von den kleinen Teilchen in das Auge des Beobachters gelangt, nimmt immer mehr $z u$, so dab man nach einer weiteren Minute das Phänomen bereits aus etwa 5 m Entfernung mit blobem Auge beobachten kann.

Die vollkommene UnregelmäBigkeit der Bewegung läht sogleich die Vermutung aufkommen, dab es sich um die Brown'sche Bewegung handelt. Die translatorische Komponente derselben kann man nicht wahrnehmen, da deren Größenordnung viel zu klein ist, um mit blobem Auge sichtbar zu sein. Wir haben es also mit der rotatorischen Komponente zu tun. Hierfür ergibt sich als alleinige Möglichkeit, daß die Teilchen winzige Kristälichen sind, 